\title{
Nanosecond Pulsed Electric Field (nsPEF) Ablation as an Alternative or Adjunct to Surgery for Treatment of Cancer
}

\author{
Ru Chen ${ }^{1,2}$, Xinhua Chen ${ }^{1,3}$ and Stephen J Beebe ${ }^{1 *}$
}

${ }^{1}$ Frank Reidy Research Center for Bioelectrics, Old Dominion University, VA 23508 USA

${ }^{2}$ Ethicon Endo Surgery, Cincinnati Ohio, USA

${ }^{3}$ Department of Hepatobiliary and Pancreatic Surgery, Department of Surgery, Zhejiang University School of Medicine, China

\begin{abstract}
Surgery as resection or transplantation remains a fundamental means for cancer treatment and often offers an opportunity for a cure. However, surgery is not always possible because of tumor proximity to blood vessels or ducts or when a patient is not healthy enough to undergo surgery. Application of nanosecond pulsed electric fields (nsPEFs) is a new approach to treat cancer using pulse power technology that was originally designed for military purposes. This novel approach deposits extremely short pulses of high power, low energy electric fields into malignant tissues using electrodes to encompass tumors. Pre-clinical studies show that treatments are effective and without local or systemic side effects, including absences of scarring. Pre-clinical trials for basal cell carcinoma are completed, but results have not been published. For treating internal tumors, electric fields can be delivered by catheter electrodes and laparoscopy procedures. Here we present a review of the literature using nsPEFs for cancer ablation and present some recent work from the author's laboratory. We demonstrate efficacy for treatment of an ectopic mouse (Hepa-16 ) and an orthotopic rat (N1-S1) Hepatocellular Carcinoma (HCC). NsPEFs eliminate tumors by mechanisms in the presence of active caspases (apoptosis) as well as in absences of active caspases (necrosis/necroptosis). Treatment also breaches small vessels, but spares larger vessels and ducts. NsPEF treatments also reduce angiogenesis as determined by decreases in Vascular Endothelia Growth Factor (VEGF). Microvascular density markers (CD-31, CD-34 and CD-105) are significantly decreased after treatment, limiting new blood vessel formation and reinforcing tumor cell demise. Furthermore, initial challenge studies show that mice are resistant to re-introduction of the same tumor cells after treatment, suggesting that nsPEFs induces immunogenic cell death and possible host cell immune responses after treatment. NSPEF ablation of cancer targets at least three hallmarks of cancer (evasion of apoptosis, angiogenesis maintenance and immune surveillance) and provides an effective alternative or adjunct therapy for cancers in skin and internal organs.
\end{abstract}

Keywords: Pulse power; Fibrosarcoma, Melanoma; Hepatocellular carcinoma; Basal cell carcinoma; Pancreatic cancer; Cancer hallmarks; Apoptosis; Caspases; Microvascular density markers

\section{Introduction}

\section{Brief highlights in the history of surgery}

Perhaps the earliest evidence of surgery came from burial sites in France $(6,500 \mathrm{BC})$ where indications of trepanation or drilling a hole in the skull were found [1]. These surgeons were nameless, but Urlugaledin $(4,000 \mathrm{BC})$ was known as a surgeon in a cradle of civilization in the Tigris - Euphrates river valley in Mesopotamia [2]. Other early noteworthy surgeons included Imhotep (circa 2650-2600 BC), an Egyptian scholar who was perhaps the first physician in early history and was considered the Egyptian god of medicine [3] and the god of science and thought [4]. He authored a medical treatise that excluded magic from medicine and contained anatomical observations, ailments, treatments and cures [5]. Sushruta (1000-600 BC), who was known as the "father of surgery", practiced in Northern India around $600 \mathrm{BC}[2]$. He authored the oldest known text in surgery, which included details of examinations, diagnoses, treatments, and prognoses of numerous ailments in addition to procedures on cosmetic surgery, plastic surgery and rhinoplasty [6]. The ancient Greeks, Herophilos and Erasistratus ( 300 BC), were the first scientists to systematically dissect human cadavers. They founded the School of Anatomy in Alexandria from which came descriptions of ligature (hemostasis), lithotomy, hernia operations, ophthalmic surgery, plastic surgery, methods of reduction of dislocations and fractures, tracheotomy, and mandrake as anesthesia [7,8]. Hua Tuo (c. 140-208) [9], an ancient Chinese physician, was the first person to perform surgery with the aid of anesthesia (mafeisan or "cannabis boil powder) about 1600 years before Europeans. Hippocrates, the "father of western medicine "founded the Hippocratic School of Medicine, which revolutionized medicine by establishing it as a distinct discipline and profession [10]. He spent 20 years in prison for differentiating medicine from religious beliefs, arguing that disease resulted from environmental factors, diet and living habits instead of punishment from gods [11]. Galen (c. 129-200) [12], another Greek, who lived in the Roman era, was a very accomplished surgeon and physician who carried out very complex surgical operations and added significantly to our understanding of surgery as well as animal and human physiology. Throughout history, surgery made regular advances in times of war. Modern surgery did not begin until three major problems were resolved. These were pain, bleeding and sepsis. Anesthetics such as ether (1500s), nitrous oxide (early 1800s) and ether-chloroform (1840s) contributed significantly to the pain problem. Louis Pasteur (1822-1895) advanced the idea that bacteria caused infections; however, this idea was slow to be accepted. Lister (1827-1912) controlled infection using antiseptic surgery. This was followed by sterilization, use of rubber gloves and antibiotics, all contributing to the problem of infections. Before the bleeding problem could be settled, physicians had to realize that loss of blood was a major problem for survival. Bleeding patients was common practice for almost every ailment for hundreds of years. When physicians finally

*Corresponding author: Stephen J Beebe, Frank Reidy Research Center for Bioelectrics, Old Dominion University, VA 23508, USA, Tel: 757-683-2405; Fax: 757451-1010; E-mail: sbeebe@odu.edu

Received March 07, 2013; Accepted March 30, 2013; Published April 10, 2013

Citation: Chen R, Chen X, Beebe SJ (2013) Nanosecond Pulsed Electric Field (nsPEF) Ablation as an Alternative or Adjunct to Surgery for Treatment of Cancer. Surgery Curr Res S12: 005. doi:10.4172/2161-1076.S12-005

Copyright: (c) 2013 Chen R, et al. This is an open-access article distributed under the terms of the Creative Commons Attribution License, which permits unrestricted use, distribution, and reproduction in any medium, provided the original author and source are credited. 
realized hemostasis was critical for survival, bleeding was controlled by point pressure, tourniquets, cauterizations and ligatures. Replacing lost blood was addressed by transfusions, but understanding concepts of blood groups was required before transfusions were successful. There are a number of claims to the first "modern" surgery. Pertinent to this review, the Mayo brothers and father, who founded the Mayo Clinic (1914), conducted one of the first surgeries in the US by removing a cancerous growth from a patient at St. Mary's Hospital (1905). Other significant advance is surgery came with the first successful kidney transplant (1954) at Peter Bent Brigham Hospital in Boston, MA [13] and the first successful heart transplant (1967) at Cape Town South Africa [14]. Other highlights in chronicles of surgery include the invention of the fiber optic endoscope (1963-64), laparoscopic or minimally invasive surgery and more recently, robotic surgery, including one or the earliest placing a needle for a brain biopsy (1985) [15] and prostate surgery (1988) [16]. Today robotics can be used in essentially all aspects of general surgery.

\section{Materials and Methods}

\section{Alternative therapies for treatment of cancer}

At the other end of these developments is the replacement of surgery or inclusion of other treatments as adjuncts to surgery for treatment of cancers. For the purposes here, an overview of the earliest examples of nsPEF treatment of cancer and then a focus on Hepato Cellular Carcinoma (HCC) with an ectopic mouse model and an orthotopic rat model. For treatment of HCC, there are a number of alternative treatments to surgery with several relatively new technologies $[17,18]$ using thermal approaches [radiofrequencies (RFA), microwaves (MWA), cryoablation] chemical-techniques (percutaneous ethanol/ acetic acid), radiological methods (transarterial chemoembolization and radioembolization, also RFA), High Frequency Ultrasound (HIFU) treatment. Importantly, there have been a number of nonthermal methods used as alternatives to surgery and many of them use local electric fields as a means of treatment. More than a quarter of a century ago, it was shown that non-thermal applications of electric fields could alter plasma membranes of cells to transport DNA across their membranes. The authors proposed that electric field interaction with lipid dipoles at local defects in the plasma membrane could cause disordered structures, leading to permeation sites for DNA entry [19]. Electro gene therapy, or gene delivery to cells or tissues, is now being used in clinical trials and has been shown to be clinically safe and effective for deliveryIL-12 to melanoma patients [20]. Another non-thermal use of electric fields came into practice in a similar way to that used for gene transfer. Instead, electroporation was used to deliver chemotherapeutic drugs such as bleomycin that were impermeable to plasma membranes [21]. This is now commonly practiced in Europe for treatment of cancer [22]. Another non-thermal approach for treating cancer is to increase electric fields such that electroporation is irreversible, thereby eliminating tumor cells by Irreversible Electroporation (IRE) of plasma membranes [23,24]. At about the same time, nanosecond pulsed electric field (nsPEF) ablation was being explored in vitro and in vivo and is the major interest in this review.

\section{Nanosecond pulsed electric field (nsPEF) ablation as a cancer treatment}

Applications of nsPEFs have been used to eliminate several tumor cell types in vitro and tumor tissues in vivo. This approach uses pulse power technology that was originally designed for military applications. It stores intense levels of electric energy and then unleashes nanosecond bursts of instantaneous power into cells or tissues. This is believed to be significant because it creates unique intracellular conditions of high power and low energy. Because the pulses are so short, when using low pulse repetition rates, the method is essentially non-thermal $[25,26]$. However, when repetition rates are higher, some heat is generated. However, if temperatures do not increase to more than $40-42^{\circ} \mathrm{C}$, the treatment can still be considered non-thermal. For example, in the first study treating B16f10 melanoma tumor in vivo, the temperature in the treated tumor rose from $28^{\circ}$ to $31^{\circ} \mathrm{C}$, clearly non-thermal [26]. In addition, ablation zones are very clearly defined based on electrode placements [27]. This allows precise treatment of tumors with appropriate margins of adjacent tissue, thereby minimizing damage to non-tumor tissue. Although there is some cell type specificity with nsPEFs $[28,29]$, all cells surrounded by electrodes and exposed to sufficiently high electric fields should be affected and undergo cell death. Not only will cancer cells be affected, but other cells will be affected including host cells that support tumor growth and slow growing cancer stem cells that are not affected by agents that target rapidly proliferating cell, will be affected. NsPEF ablation induces caspase-dependent and caspase-independent programmed cell death [30], at least in part, by inducing supra-electroporation [31,32], which consists of high density nanopores, not only in plasma membranes but also in organelle membranes and possibly other mechanisms such as effects on voltage gated channels [33] and perhaps on proteins $[34,35]$. The effect on intracellular membranes is significant because permeabilization of plasma membranes is not always sufficient for cell death induction under nsPEF conditions [35]. While poration of plasma membranes and intracellular stores is critical for elevating intracellular calcium, effects on mitochondria membranes and/or channels and the mitochondria membrane potential $(\Delta \Psi \mathrm{m})$ appear to be required for nsPEF-induced cell death, at least under certain conditions [35]. Thus, by targeting plasma membranes, $\Delta \Psi \mathrm{m}$, and/or membranes surrounding intracellular calcium stores, applications of nsPEFs affect multiple therapeutic targets and cancer hallmarks [36].

\section{Treating ectopic mouse B10.2 fibrosarcomatumors with nsPEFs}

The first application of nsPEFs for cancer treatment was using B10.2 fibrosarcoma cells in vitro and in a mouse tumor xenograft model [37]. One of the major points was showing that nsPEFs differed from conventional electroporation. In so doing, in vitro studies exposing cells to nsPEFs in cuvettes demonstrated that nsPEFs induced apoptosis markers including the presence of active caspases and phosphatidylserine externalization, which was most likely due to direct electric field effects on plasma membranes [38]. In tissues removed from ectopic tumors and treated ex vivo in cuvettes, a 6-fold increase in TUNEL positive cells was observed compared to sham treated controls 5 hours after treatment with nsPEFs. Mouse B10.2 tumors were also treated in vivo. These treatments primarily served as a proof of principle that nsPEFs could have a deleterious effect on tumors. Tumor cells were implanted in each flank of mice. One tumor served as a sham control and the contra lateral tumor was treated. Since these were the first in vivo studies carried out with nsPEFs, there were no guidelines to choose treatment conditions. After tumors reached about 5-8 mm in diameter as measured by calipers (day 0 ), tumors were exposed to two pulses with durations of $300 \mathrm{~ns}$ and electric fields at $75 \mathrm{kV} / \mathrm{cm}$ and $1 \mathrm{~Hz}$ on day 2 and 5 pulses under the same conditions on day 5 using a two needle electrode. In 5 animals, treated tumors grew $62 \%$ slower than sham controls. In three other animals, treated tumors were $60 \%$ smaller by weight than sham controls. It is now known that these treatment conditions were far less rigorous than needed for tumor elimination based on results from treatment of ectopic mouse B16f10 
melanoma, ectopic mouse Hepa1-6 HCC and orthotopic rat N1S1 tumors (see below). Nevertheless, these experiments verified that nsPEFs could induce apoptosis in vitro and ex vivo and significantly reduce tumor growth in vivo. In studies that followed with Jurkat cells in vitro, it was shown that nsPEFs induced cytochrome $c$ release and activated caspase- 3 catalytic activity, confirming that apoptosis was at least one of the cell death pathways induced by nsPEFs [39]. A later paper demonstrated the presence of caspase-dependent as well as caspase-independent activities in Jurkat cells [30].

\section{Treating mouse B16f10 melanoma tumors in vivo with nsPEFs}

Later, nsPEFs were shown to eliminate mouse B16f10 tumor in a mouse ectopic model without recurrence under optimal conditions [26]. In these studies two different delivery electrode designs were tested including a 5 needle array and two parallel plates. When the 5 needle array was used, the electric fields were heterogeneous with the highest field at the center needle and field lines parallel to the surface of the skin. Treated tumors shrink by about $75 \%$ within 8 days when given two treatments with 100 pulses with durations of $300 \mathrm{~ns}$ and electric fields at $20 \mathrm{kV} / \mathrm{cm}$ and $0.5 \mathrm{~Hz}$. Tumor responses were dependent on electric fields and pulse numbers. After pulsing, blood flow to tumors was interrupted and did not recover for about two weeks. In addition to tumor damage, the stratum corneum was damaged, showing signs of necrosis and hemorrhage. Interestingly, this was not a thermal injury or a burn, which would be expressed immediately; the temperature increase was only $3^{\circ} \mathrm{C}$, significantly lower than required for a hyperthermic injury. Thus, this is more likely an electrical injury, perhaps due to effects on proteins caused by dissociation of ionizable side groups, reorientation of peptide dipoles within the protein in the direction of the electric field, conformational changes and denaturation [40]. Using the plate electrode design, tumors were positioned between parallel plates. This provided a more homogeneous electric field, which was oriented perpendicular to the skin surface instead of parallel to it, like that with needle electrodes. In this configuration, the surface of the skin was between electrodes and the tumor. Using four 100 pulse treatments ( 3 on day 0 and 1 on day 3 ) and electric fields of $40 \mathrm{kV} / \mathrm{cm}$, tumors shrank by $90 \%$ in two weeks. The skin responded with a black scab on the stratum corneum, composed of clotted red blood cells, which remained for about two weeks during regeneration of the stratum corneum. After about two weeks of regression, all tumors began to grow again. When tumors began to grow again and were given additional treatments including a 3 day series of 100 pulses, a complete remission was observed for as long as two months. Two immediate changes after nsPEF treatment were hypothesized to cause tumor regression. One was a rapid pyknosis of tumor nuclei and the other a cession of blood flow to tumors. Within the first few minutes, tumor cell nuclei shrank by $54 \%$ and within 3 hours they shrank by $68 \%$. The absence of blood flow to tumors within the first 15 minutes after treatment was indicated by trans-illumination and power Doppler ultrasound reconstructions. As long as blood flow did not return to treatment areas, tumors did not return; however, if blood flow returned with two weeks after treatment, tumor growth returned. In addition to killing tumor cells, as indicated by pyknotic tumor cell nuclei, the absence of blood flow appears to be an important factor to tumor regression. In later studies, it was shown that B16f10-eGFP tumors could be eliminated in a single treatment using 2000 pulse with duration of $100 \mathrm{~ns}$ at $30 \mathrm{kV} / \mathrm{cm}$ at $5-7$ $\mathrm{Hz}$ [41]. These studies differ from the previous one in several ways. This study used $\mathrm{Nu} / \mathrm{Nu}$ mice, which are immunodeficient, instead of $\mathrm{SKH}$ 1 mice, which have a relative strong immune system. The treatments were with shorter pulse durations (100 ns instead of $300 \mathrm{~ns}$ ) and higher repetition rates $(5-7 \mathrm{~Hz}$ instead of $0.5 \mathrm{~Hz})$. The higher repetition rate caused temperatures to increase to about $40^{\circ} \mathrm{C}$, yet the treatment is still considered non-thermal. Another difference included using suction electrodes, which were compatible for human use. This design limited the treatment volume to tissues that could be placed between electrodes within the suction cup. The skin was also affected by this nsPEF treatment; however, scabs that formed fell off by the end of the second week, leaving a coloration difference from untreated skin that faded leaving no scar. In unpublished clinical trials, nsPEFs affected human skin causing only mild irritation, erythema and itching leaving some discoloration that faded and no scar [42]. Thus, an important advantage and benefit for nsPEF treatment of skin cancer over surgery is the absence of scarring after treatment. Effective ablation was achieved in radiation-induced murine Basal Cell Carcinomas (BCC), which was similar to human BCC, in Ptch $1^{+/} \mathrm{K} 14-\mathrm{Cre}-\mathrm{ERp} 53 \mathrm{fl} / \mathrm{fl}$ mice using a 6-post dual configuration suction electrode, which was the best of three electrode designs tested. The electrode configuration and complete encapsulation of the tumors were critical components for complete ablation in a single treatment. Using 2700 pulses with $100 \mathrm{~ns}$ durations and electric fields of $30 \mathrm{kV} / \mathrm{cm}$ at $5-7 \mathrm{~Hz}, \mathrm{BCC}$ tumors shrank $76 \%$ at 2 weeks and $99.9 \%$ at four weeks after treatment [43]. Complete ablation without recurrence of human pancreatic carcinoma was also demonstrated in a murine xenograft model using similar conditions, but with 500-1000 pulses showing good efficacy [44].

\section{The devastation of Hepatocellular Carcinoma}

Liver cancer is the third most common cause of mortality worldwide [WHO statistics] and Hepato Cellular Carcinoma (HCC) is the most common, occurring in $75 \%$ of all liver cancers [45]. Africa and Southeast Asia have highest incidences of HCC and it is the most common cancer in Japan. The incidence and mortality rates of HCC are nearly equal because the fatality rates are so high. The prognosis for HCC is usually poor because symptoms and diagnosis do not generally occur until advanced stages. The incidence and mortality rates have increased recently in the US and are affecting younger persons, mostly due to increased infections with hepatitis $C$ and $B$ viruses [46]. While the prognosis of cancer patients is generally determined by tumor size, HCC treatment efficacy is also determined by underlying diseases such as cirrhosis or other functional maladies [47]. These underlying diseases, in part, account for poor responses to chemotherapeutic agents and ionizing radiation and also account for $<20 \%$ of patient eligibility for resection. Still, several new developments have led to increases in early diagnoses including greater awareness of the disease, new screening approaches and more definitive diagnosis using highresolution imaging of the liver [48]. Yet, a major remaining barrier is the absence of successful management strategies that avoid multiple treatments and prevent recurrences. One of the most comprehensive classification system used for HCC in the US is the Barcelona-Clinic Liver Cancer (BCLC) staging classification and treatment schedule $[49,50]$. The system includes 5 tumor staging categories that consider size and number of nodules, the degree of liver function and the presence or absence of metastasis. They include very early $(\mathrm{O})$, early (A), intermediate (B), advanced (C) and terminal (D) stages. Stages $\mathrm{O}$, $\mathrm{A}$ and $\mathrm{B}$ are treated with resection, liver transplantation, percutaneous ethanol (or acetic acid), radiofrequency ablation or Transcatheter Arterial Chemoembolization (TACE). Advanced and terminal stages are generally treated with palliative measures. HCCs are generally resistant to chemotherapeutic agents and radiation. For example, sorafenib is a new oral multi-kinase inhibitor for advanced HCC with modest clinical efficacy extending survival by only a few months [51,52]. New treatment strategies are needed for stages $\mathrm{O}, \mathrm{A}$ and $\mathrm{B}$ 
that avoid multiple procedures and recurrences. This is a niche that can be filled using pulse power technology with nanosecond pulsed electric field (nsPEF) ablation of HCCs. Hepatocytes are the major liver cell type and are vulnerable to most forms of liver injury either as primary or secondary insults. Continuous cell turnover by apoptosis, which is tightly coupled to inflammation and fibrosis [53-55], leads to activation of myofibroblasts, hepatic fibrosis, cirrhosis and HCC [56]. Such chronic pro-apoptotic pressure provides the basis for cancerrelated mutations and promotes development of apoptosis evasion [57], a hallmark of cancer [36]. Production of pro-inflammatory products from macrophages, stellate cells and Kupffer cells during phagocytosis of apoptotic bodies appears to be responsible for the link between apoptosis and fibrosis [53,57,58], a paradigm that appears to be unique to hepatocyte apoptosis. Cancer also emerges when immunological control fails and transformed cells develop resistance against cell death signaling pathways. The same mechanisms underlie the poor responsiveness of HCC towards chemotherapy. Mechanisms of cell death exhibit complexity beyond apoptosis and necrosis as cross-talk among signaling pathways involved in cancer hallmarks makes clear. Also the tumor microenvironment has an impact on cell signaling and cell death. While significant progress has been made in understanding tumorigenesis and cancer molecular biology, the overall therapeutic effects on cancer mortality have been disappointing. While the primary pathways that drive cancer are known and a significant number of drugs targeted for these pathways have been developed and tested, virtually none of these drugs or treatments has provided a cure. The scientific community is conceptually reexamining approaches for cancer treatment, considering cancer as manifestations of eight essential hallmarks [36] and instead of conceiving cancer as a foreign invader [59], reevaluating cancer as a criminal gang (the cancer) within the local community (cancer microenvironment) that coerces the local population (supporting host cells, blood vessels) to use their resources (growth, differentiation and vessel-forming factors) to support the gang's criminal activity (the tumor as an organ).

\section{Treating ectopic mouse Hepa1-6 HCC tumors in vivo with nsPEFs}

As a first step to address the possibility of using nsPEFs for treating HCC, an ectopic mouse Hep1-6 HCC tumor model was established in C57BL/6 mice and optimal conditions were determined as well as cell death mechanisms in response to nsPEF [60]. In this study a number of different conditions were tested. Pulses with durations of 30 or 100 ns and fast rise times were delivered by an electrode with a5 needle, $4+1$ configuration or an electrode with a center needle and a solid ring surrounding it. The electric field distribution in both electrode configurations were heterogeneous with electric fields as high a 230 $\mathrm{kV} / \mathrm{cm}$ at the center needle with electric fields decreasing towards the ground electrodes, where electric fields were on the order of $40-90 \mathrm{kV} /$ $\mathrm{cm}$ [60]. Different electric field strengths $(33,50$ and $68 \mathrm{kV} / \mathrm{cm})$ were tested with 900 pulses delivered at $1 \mathrm{~Hz}$ as a single treatment or as 3 separate treatments on days 1, 3 and 5 with 300 pulses each. The mice were followed for 269 days after treatment. For those mice that survived after treatment, this is a significant cancer free span considering this is approximately half of a mouse's life expectancy. If this would translate to humans with an average life expectancy of 80 years and ignoring differences in cancer growth rates, which varies significantly among cancers, an individual who was diagnosed with cancer and successfully treated by the age of 40 could live cancer free of the rest of their life. This is significant since with optimal treatment conditions, $75 \%$ of treated animals survived this long when the experiment was terminated. In these studies, optimal treatments with $75 \%$ survival include 900 pulses either delivered at one time or 3 times with 300 pulses each on alternate days, with durations of $100 \mathrm{~ns}$ and electric fields at $65 \mathrm{kV} / \mathrm{cm}$ and $1 \mathrm{~Hz}$. Generally, after tumors were treated, they stabilized or grew slightly larger until the third day when they decreased in size and were nondetectable by 14-21 days. Control, sham treated tumors continued to grow for about 14-18 days when mice were humanely euthanized due to tumor burden. When pulse durations were decreased to $30 \mathrm{~ns}$ or electric fields were decreased to 33 or $50 \mathrm{kV} / \mathrm{cm}$, treatment efficacies were significantly lower. For those tumors that were treated under optimal conditions, the tumor cell nuclei rapidly decreased in size during the first hour. Tumor cell nuclei featured highly condensed chromatin, segregating into sharply defined bodies (nuclear pyknosis) by 3-6 hours after treatment. Fifty to sixty percent of treated tumor nuclei were TUNEL positive by 3 hours after treatment. The number of TUNEL positive cells decreased thereafter over the next 12-24 hours. The presence of active executioner caspases, as identified by specific antibodies, followed a similar time course after treatment. The presence of active caspase- $3,-6$ and -7 peaked at 3 hours and decreased thereafter over the next 5 hours. Active caspase- $3,-6$ and -7 activity was present in about $45-50 \%, 12-18 \%$ and $20-40 \%$ of cells, respectively, 3 hours after treatment at their peak. Activities of capase- 3 and -6 fell off rapidly between 4-8 hours after treatment but caspase- 7 activity fell at a slower rate. Since these analyses were carried our separately, it is not possible to know whether a given cell exhibited more than one or more than one active caspase or whether there were cell type specificity for specific caspases. Nevertheless, it is likely that not all cells exhibit active caspases and are undergoing caspase-independent cell death. Since after efficacious treatment all tumors disappear, there are few or no cells that do not undergo cell death. Another indicator of apoptosis was the absence of phosphorylated Bad in effectively treated Hep1-6 tumors. When Bad is phosphorylated, it is bound to the protein 14-3-3, which removes it from possible heterodimerization with Bcl-xl or Bcl2, which neutralizes their protective effect on mitochondria thereby promoting survival [61]. When Bad is in the un-phosphorylated form, which was present in treated Hep1-6 tumor cells, it heterodimerizes with $\mathrm{Bcl}-\mathrm{xl}$ or $\mathrm{Bcl}-2$, thereby removing their protective effect promoting cell death. Therefore, the presence of active caspases and the absence of phosphorylated Bad are conditions that promote cell death by apoptosis. It was previously shown that nsPEFs had significant effects on tumor vascularity in B16f10 melanoma tumors in vivo [62]. In these studies, it was shown that there were progressive decreases during 21 days after treatment in Vascular Endothelial Cell Factor (VEGF), the most ubiquitous pro-angiogenic factor, a requirement for the angiogenic switch [63], a limiting factor for multistage carcinogenesis [36] and Platelet Derived Endothelial Growth Factor (PD-ECGF). In addition there were progressive decreases over this same time period for several micro vascular density factors, including CD-31, CD-34 and CD-105. CD-31 (PECAM-1), a platelet-endothelial cell adhesion molecule used as a pan-endothelial cell marker, and CD-34, an endothelial cell marker, were decreased by $65-70 \%$. CD-105 (endoglin) was decreased $>40 \%$. CD-105, which is part of the TGF $\beta$ receptor complex, is an important angiogenic factor that is strongly expressed in tumors and is an independent prognostic indicator, wherein increased MVD correlates with shorter survival [64].

\section{Does nsPEF treatment induce an immune response in ectopic mouse Hep 1-6 HCC tumors?}

Using this same mouse Hep1-6 model in C57BL6 mice, we decided to challenge mice with a second injection in the opposite flank of the same Hep 1-6 tumors cells after the primary tumor had been successfully cleared for 60 days [65]. Age match, naïve control mice were also 
injected in the same way. When Hepa 1-6 tumors were successfully clear after treatment with treatments with 900 pulses at 100 ns and 55 $\mathrm{kV} / \mathrm{cm}$ in 6 of 8 treated mice, tumors cells were injected in the opposite flank as before. None of those 6 mice that were successfully treated before grew tumors for 49 days before the experiment was terminated. In naïve age-match control HCC tumors grew to treatable sizes in less than two weeks. These results suggest that nsPEF ablation allows a host cell immune response. While these studies must be repeated and the mechanisms of this resistance further investigated, these results suggest that nsPEF ablation addresses another cancer hallmark, evasion of immune surveillance. Another study compared treatments with nsPEFs and surgical removal of B16f10-GFP in melanoma in SKH-1 mice [66]. After the respective treatments, challenge injections if the same cells grew much slower in nsPEF-treated mice that those mice treated with surgery. In addition, CD4+ T-cells were present in treated tumors as well as tumors that had not been treated in mice with treated melanomas. These results suggest than nsPEF-stimulated tumor cell death induces an immune response. The results from the Hep1-6 in vivo studies indicated that nsPEFs act on several cancer hallmarks [36]. These include resisting cell death by apoptosis, resisting angiogenesis (or anti-vascular activities) $[60,62]$ and perhaps overcoming evasion of immune surveillance $[65,66]$.

\section{Treating orthotopic rat N1-S1 HCC tumors in vivo with} nsPEFs

In more recent studies, we have established and tested an orthotopic rat N1-S1 HCC using conditions similar to those in the Hep1-6 mouse model discussed above. The studies carried out previously demonstrated that nsPEF could effectively eliminate tumors when they were implanted under the skin. We wanted to extend applications of nsPEFs to treating internal tumors. Given successes with the ectopic mouse HCC model and the "HCC problem", which is increasing in the US and already a major problem worldwide, we pursued application of nsPEF to HCC tumors implanted in the liver of rats. We chose a rat model because of the larger liver and the need to carry out two surgeries and later three surgeries to demonstrate efficacy of nsPEFs. Thus, these studies combine nsPEF treatment with laparotomy as a proof or principle, before establishing treatments with laparoscopy and catheter electrodes. Thus, in this model, we introduced N1-S1-Luc cells under the liver capsule through a small incision in the abdominal wall. N1-S1 cells were stably transfected with a luciferase gene downstream of a CMV promoter. When tumors grew to about $0.5 \mathrm{~cm}$, we exposed the liver again and treated with nsPEFs before closing. Tumor growth was monitored by luciferase activity (luminescence) ultrasound. In this model, we used a 5 needle $(4+1)$ array [60] and treated tumors using pulses with electric fields at $50 \mathrm{kV} / \mathrm{cm}$ and delivered at $1 \mathrm{~Hz}$ and pulse durations of $100 \mathrm{~ns}$ with a rise-fall time of $10 \mathrm{~ns}$. A study was carried out testing 100, 300, 500 or 1000 pulses. Fourteen days after treatment, rats were humanely euthanized and tumor sizes were determined.

\section{Analysis of HCC tumors by size and bioluminescence after nsPEF treatment}

Figure 1 shows results indicating tumor sizes were decreased with all pulse numbers. Using 100 pulses tumor sizes were decreased but were only significantly less than sham-treated controls 14 days after treatment. Tumor sizes were smaller using 300, 500 and 1000 pulses with the later condition decreasing tumor size about 6 -fold. In subsequent experiments, when treated tumors were measured 6 weeks later, the 100 and 300 pulse conditions were insufficient to eliminate tumors; tumors had continued growing slowly between 2 and 6 weeks after treatment. We had initiated tumor development using an N1-S1 clone stably transfected with luciferase to monitor tumor growth by luminescence. Figure 2 and table 1 shows tumor luminescent images of the same rats taken in the IVIS 100 in a sham-treated control and a rat treated with 1000 pulses with durations of $100 \mathrm{~ns}$ and electric fields at $50 \mathrm{kV} / \mathrm{cm}$ delivered at $1 \mathrm{~Hz}$. Luminescent images were determined on the day of treatment (day 0 ) and 7 and 10 days after treatment. The table below the image indicates luminescence values and tumor sizes based on luminescence. Tumors from one sham-treated and one typical nsPEF- treated rat are shown as examples. It can be seen that by day 7 the sham treated tumor grew 5-6-fold compared to the size just before treatment and continued to grow for 10 days. In the treated rat, there was no detectable luminescence, indicating that treated tumors were absent 7-10 days after treatment. In order to evaluate longer term efficacy of nsPEFs, we treated tumors and evaluated the presence or absence of tumors seven weeks post-treatment. Figure 3 shows a Kaplan-Meier survival curve [67] for one experimental series with 7 rats that were sham treated and 8 rats that were treated with 1000 pulses with durations of $100 \mathrm{~ns}$ and electric fields at $50 \mathrm{kV} / \mathrm{cm}$ and 1

A.

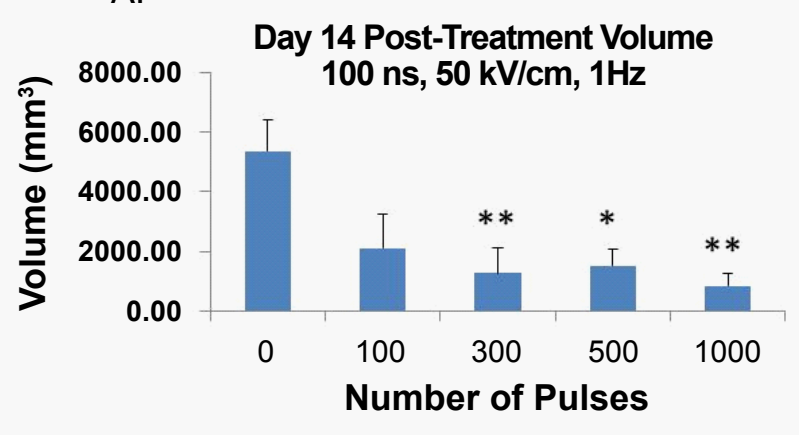

B.

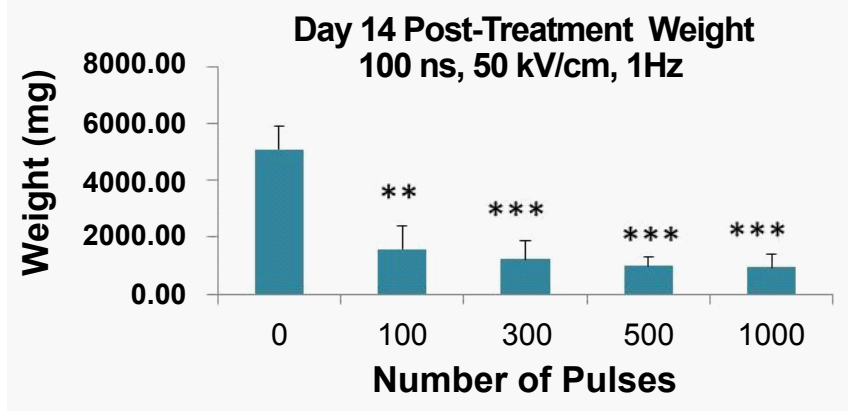

Figure 1: N1-S1-Luc HCC tumor sizes 14 days post-nsPEFs treatment. - N1$\mathrm{S} 1$ cells were transfected with the gWiz-Luc plasmid driven by the CMV IE promoter (Aldevron, Madison $\mathrm{WI}$ ). Clones were isolated as stable transfectant and the clone with the highest expression level was used. A liver lobe from Sprague Dawley rats (Harlan, Frederick MD) was exposed through a small incision in the abdomen and rat N1-S1-Luc HCC cells $\left(1 \times 10^{6}\right)$ (N1-S1 from ATCC) were injected under the liver capsule. Seven days later, the liver lobe was again exposed though an abdominal incision and tumors $(\sim 0.5$ $\mathrm{cm}$ in diameter) were treated with various pulse numbers with durations of $100 \mathrm{~ns}$, electric fields of $50 \mathrm{kV} / \mathrm{cm}$ at $1 \mathrm{~Hz}$ ( $\mathrm{n}=8$ for all groups). Rats were humanely euthanized 14 days after treatment. Treated lesions were excised to determine volume $(A)$ and weight $(B)$. Tumor volumes were determined using the formula for prolatespheroids (square of the width $x$ length $\times 0.52$ ): $V$ $=0.52 \times(D 1 \times D 2) \times 2$, where D1 and D2 are short and long tumor diameters, respectively. The symbols "*” indicates statistical significance compared with the zero pulse group. $P$ values were calculated using a one-way ANOVA followed by Tukey tests $\left({ }^{*} p<0.05,{ }^{* *} p<0.01{ }^{* * *} p<0.001\right)$. 


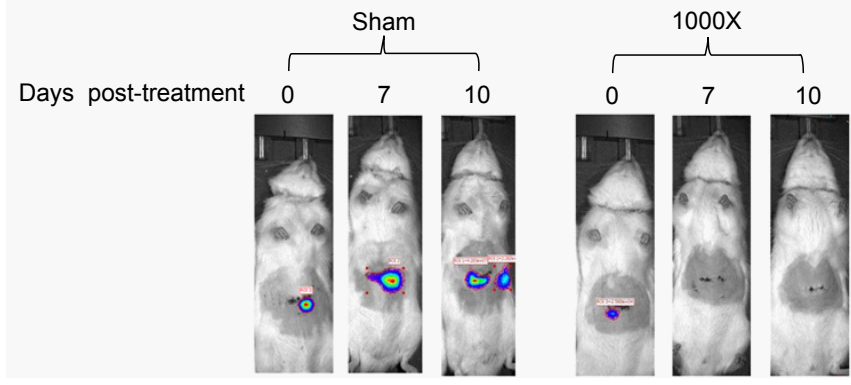

Figure 2: NsPEFs eliminate N1-S1-Luc tumors determined by luminescence 7-10 days after treatment. -Tumors were initiated as indicated in the legend to Figure 1. Seven days after initiation tumors were treated with 1000 pulses (1000X) with durations of $100 \mathrm{~ns}$ and electric fields at $50 \mathrm{kV} / \mathrm{cm}$ at $1 \mathrm{~Hz}$. On the day of treatment (day 0 ) and 7 and 10 days after treatment tumor bioluminescence was determined on the same animals using non-invasive in vivo imaging with the IVIS Spectrum (Caliper Life Sciences, Xenogene). One sham-treated tumor and one typical rat with a treated tumor are shown.

\begin{tabular}{|c|c|c|c|c|}
\hline & Days post-treatment & $\mathbf{0}$ days & $\mathbf{7}$ days & $\mathbf{1 0}$ days \\
\hline \multirow{2}{*}{ Sham } & Luminescence $(\mathrm{p} / \mathrm{s})$ & $9.47 \times 10^{6}$ & $5.40 \times 10^{7}$ & $4.28 \times 10^{7}+2.28 \times 10^{7}$ \\
\cline { 2 - 5 } & Area $\left(\mathrm{cm}^{2}\right)$ & 0.545 & 2.58 & $2.92+2.25$ \\
\hline \multirow{2}{*}{$\begin{array}{c}1000 \mathrm{x} \\
\text { nsPEFs }\end{array}$} & Luminescence $(\mathrm{p} / \mathrm{s})$ & $1.54 \times 10^{7}$ & 0 & 0 \\
\cline { 2 - 5 } & Area $\left(\mathrm{cm}^{2}\right)$ & 0.957 & 0 & 0 \\
\hline
\end{tabular}

Table 1: The table below indicates the luminescence units and tumor area in $\mathrm{cm}^{2}$. The designation 1000X indicates that tumors were pulsed 1000 times.

$\mathrm{Hz}$. The results show that the all sham-treated rats had to be humanely euthanized by 25 days after tumor initiation because of tumor burden $(1.5-2.0 \mathrm{~cm})$ according to our IACUC protocol. All 8 rats treated with nsPEFs remained alive and tumor-free for at least 49 days. When tumor size was checked after humane euthanasia or when rats were exposed to a second challenge injection of N1-S1 tumor cells, treated tumors were complete absent and liver tissue had returned to normal hepatic structure in the treated area.

\section{Effect of nsPEF treatment on liver blood flow}

It was previously demonstrated that nsPEFs had significant effects on blood flow in the areas of treated ectopic B16f10 [26,62] and Hepa16 HCC tumors [60]. While this was advantageous to inhibit ectopic tumor growth, we were interested to evaluate post-treatment liver blood flow, which is heavily vascularized. Effects on liver blood flow are important in HCC treatment since it is common for HCC patients to have compromised liver function. Figure 4 shows blood flow analysis by laser Doppler before, immediately after and 7 days after treatment. Immediately after treatment, blood flow was decreased by $50-60 \%$ in the treated liver area. Only the treated area was affected. However, 7 days later, blood flow had returned to normal and tended to be greater than the original control. This suggests that in nsPEF treated liver, effects on blood flow or anti-vascular effects or effects on angiogenesis, which occurred in ectopic tumors, is not likely to be a factor or a cancer hallmark in nsPEF treated HCC demise. Nevertheless, nsPEF treatments are an effective therapy for HCC.

\section{Histological examination of normal liver and sham and HCC treated tissue}

In order to determine effects of nsPEFs on treated N1-S1 HCC tumor tissue and compare it to normal liver and sham-treated control tumor tissue, we collected samples two weeks post-treatment from each of these tissues and prepared them for H\&E staining and microscopy (Figure 5). The image of normal control liver shows a central vein and normal lobular hepatic architecture with cellular cords radiating from it with asymmetrical sinusoids and a typical scattering of collagen. In sham treated N1-S1, cancer cells can be seen infiltrating normal hepatic tissue with globular-like structures and sparse nuclei. Leukocyte infiltration in peripheral areas is evident. The vascular structures containing red cells are irregular and more numerous than in normal liver. In nsPEF-treated N1-S1 HCC, the cancerous tissues is completely destroyed with an absence of globular cancer cells and an enormous infiltration of leukocytes. We more-or-less expected this massive leukocyte infiltration given our previous results in Hepa1-6 HCC treated tumors. We had previously observed that after successfully treating Hepa1-6 HCC mouse tumors with nsPEFs (1000 pulses, 100

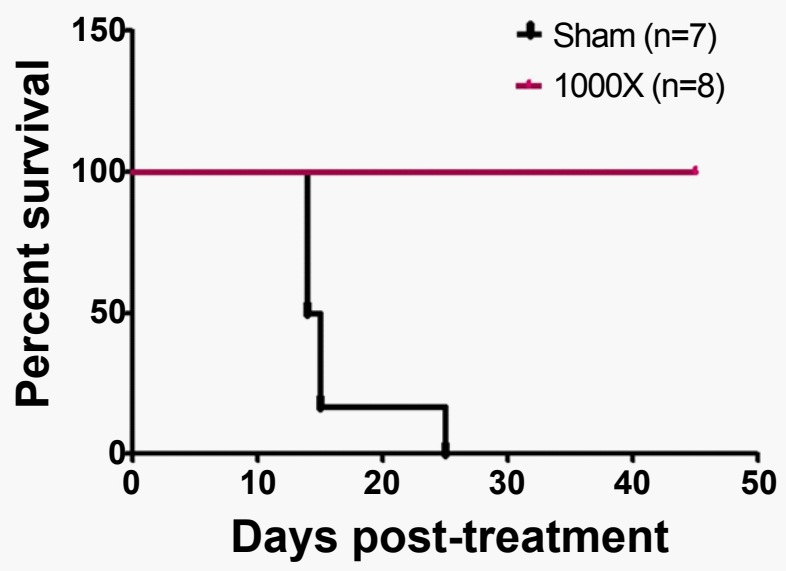

Figure 3: NsPEFs eliminate tumors for as long as 7 weeks. - N1-S1-Luc tumors were initiated and treated with 1000 pulses with 100 ns durations and $50 \mathrm{kV} / \mathrm{cm}$ at $1 \mathrm{~Hz}$. In this typical trial, 7 sham and 8 treated rats were treated. After treatment, tumors were followed and analyzed by ultrasound. When sham treated tumors reach $1.5 \mathrm{~cm}$, they were humanely euthanized according to our IACUC approved protocol. Forty nine days after treatment the experiment was terminated and animals were humanely euthanized. All surviving animals had been treated and were tumor free. The designation 1000X indicates that tumors were pulsed 1000 times.

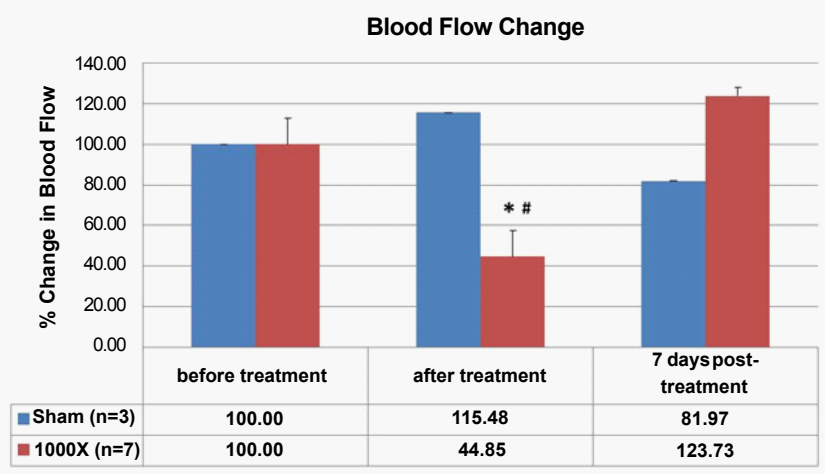

Figure 4: Blood flow to the treated tumor area is transiently reduced. - N1S1-Luc tumors were initiated and treated as describe in the legend to Figure 1. Before, immediately after and 7 days after treatment, Liver blood flow in the treated area was determine by laser Doppler imaging (Moor LDLS laser Doppler imager) and expressed as perfusion units. The data are expressed as percent of blood flow immediately before treatment. The * indicates the change is significant compare with pre-treatment (one way-ANOVA, $p<0.01$ ) \# indicates the two post-treatment groups are statistically different (two wayANOVA, $p<0.001$ ). The designation $1000 \mathrm{X}$ indicates that tumors were pulsed 1000 times. 


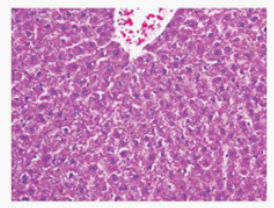

Normal Liver

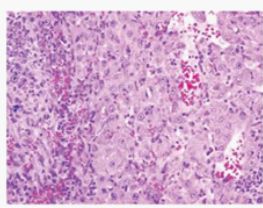

Sham

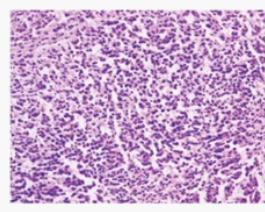

1000 pulses
Figure 5: NsPEF treatment destroys HCC tumors and induces infiltration of immune cells. - N1-S1-Luc HCC tumors were initiated as described in the legend to Figure 1 and treated as described in the legend to Figure 2. Then, 14 days after treatment rats were humanely euthanized and liver tissue was taken from normal liver removed from a distant untreated lobe, from shamtreated HCC tumor and from HCC tumor treated with nsPEFs. Tissues were washed in PBS, fixed in $10 \%$ Neutral Buffered Formalin (NBF), followed by paraffin embedded, sectioning and H\&E staining. Images were visualized using a Nikon Eclipse 50i microscope (Nikon, Tokyo, Japan) equipped with a 10X/0.25 numerical aperture, and acquired using a Go-5 camera (QImaging, Surrey, Canada) and QCapture Pro 6.0 software.

ns, $55-60 \mathrm{kV} / \mathrm{cm}$ at $1 \mathrm{~Hz}$ ) (6 out of 8 mice), challenge injections of the same tumor cells in the opposite flank 49 days later failed to grow tumors ( 6 out of 6 mice), while age-matched, naïve control mice readily grew tumors ( 6 out of 6 mice) [65]. These results are under continuing investigation to determine more specifically if an immune response is present in the orthotopic N1-S1 HCC model.

\section{Does nsPEF treatment induce an immune response in orthotopic rat N1-S1 HCC tumors?}

In ongoing studies, rats with successfully treated tumors have been challenged with a second injection or a challenge injection of the same N1-S1 cells that initiated original tumors. In these preliminary studies the challenged tumors do not grow while naïve age-matched control rats grow tumors like those tumors in sham treated control rats. This suggests that, like response to challenge in ectopic Hepa1-6 in mice, response are similar in rats, indicating that this phenomena is not species specific, is evident in an orthotopic HCC model and not confined to tumors originating in skin. The absence of challenge-tumor cell growth after successful nsPEF treatment of mouse Hepa1-6 and rat N1-S1 HCC tumors suggests the presence of immunogenic cell death. These results also indicate that these nsPEF-induced challenge phenomena are not species-specific. The mechanisms for immunogenic cell death are becoming clearer; however this clarity is complex. It is now known that physiological cell death, which is immunologically silent or tolerogenic, and cancer cell death, which can be immunogenic, are perceived differently by the immune system [68]. Thus, there must be mechanisms that differentiate among types of cell death [69]. Some (doxorubicin, cyclophosphamide, oxaliplatin) but not all apoptotic stimuli can induce immunogenic cell death, suggesting biochemical heterogeneity within apoptosis mechanisms. Those chemotherapeutic agents that do induce immunity depend on it for at least part of their efficacy $[70,71]$. There is also evidence that cells that experience stress responses and enter autophagy before undergoing apoptosis, necrosis or secondary necrosis may exhibit a more robust immunologic cell death. Induction of senescence by p53 in HCC can stimulate a robust anti-tumor response [69]. There are a number of factors that work together that determine whether cell death is immunogenic or not. These include the "history" of the cell, such as activation state or stress responses; the nature of the cell death stimulus; the cell death pathway and the availability of competent immune cells to carry out the response [69]. It appears that the pre-apoptotic exposure to calreticulin and the late apoptotic or secondary necrotic secretion of high mobility group box 1 (HMGB1), which acts on the toll-like receptor- 4 on dendritic cells, stimulates optimal antigen processing [68,72]. It will be interesting to determine whether nsPEFs exhibit some of the characteristics of immunogenic cell death. Such responses would provide significant advantage to prevent tumor recurrence and possibly vaccinate against HCC tumors that express the same antigen(s).

\section{NsPEFs combined with other treatment modalities}

NsPEF ablation impacts several well-characterized cancer therapeutic targets. It has shown success as a substitute for drug therapy and can be used in combination with other treatments; multiple therapies are common in essentially all cancer treatments and have been tried with nsPEFs [73]. In studies using concurrent treatments with low concentrations of gemcitabine and nsPEFs in mouse Cal 27 squamous cell carcinoma cells, synergistic activities were observed compared to summation of both treatment alone for inhibiting cell proliferation and inducing apoptosis and necrosis. The synergistic response was non-specific, or atleast not universal, since no synergism for cell invasion was observed with combined treatments. While these experiments were designed to reduce tumor burden with nsPEFs before chemotherapy, other strategies could include sensitizing tumors with a chemotherapeutic agent(s) before treating with nsPEFs or reducing tumor burden with nsPEFs before resection.

\section{Summary of cancer treatment with nsPEFs}

NsPEF ablation therapy has a number of advantages for anti-tumor effects. They have several therapeutic targets, which are included in a single treatment as opposed to treating cancer with individual agents that affect evasion of apoptosis, sustained angiogenesis (or antivascular), and evasion of immune responses. The anti-vascular effect may be limited to tumors implanted or developed in the skin; however, additional studies of vascular effects in highly perfused liver warrant further investigation. The evasion of immune responses assumes the latter response is supported by further testing with nsPEFs in vivo. Certainly this last response will provide a substantial benefit for cancer treatment. Furthermore, nsPEFs induce cell death by caspase-dependent and -independent mechanisms [30] by targeting mitochondria and plasma membranes for calcium influx [35]. The heterogeneity of these effects is meaningful because nsPEFs can bypass a number of possible cancer mutations when tumor cells are adequately treated as shown here for treating N1-S1 HCC. For example, since nsPEFs can induce caspase-independent cell death, oncogenic mechanisms that evade apoptosis can be superseded. In contrast, the treatment itself is well defined by placement of electrodes to surround the tumor [27]. This stipulates specificity for tumor tissue and surrounding margins to minimize damage to surrounding non-cancerous tissues. When electric fields are sufficiently intense within this treatment zone, all cells can be killed, including tumor cells and host cells that provide needed growth and angiogenic factors for tumor growth, sustenance and metastasis. Given that nsPEFs can eliminate cells regardless of their proliferation rate, and in fact require less intense conditions (fewer pulses or lower electric fields) for cells that are not proliferating (are not in the S-phase) [74], it is likely that when electric fields are high enough, nsPEFs can also eliminate slowly dividing cancer stem cells, which could eliminate one possible cause of recurrences of disease, which can occur with agents that only affect rapidly proliferating cells. For treatment of skin tumors, nsPEF do not appear to leave scars after treatment in mice [26] or humans [42]. This will have valuable significance for individuals with skin cancer. Finally, there is an absence of local or systemic side effects with nsPEF treatment, providing an improvement over treatment with chemotherapeutic agents and ionizing radiation. When 
these agents would be used in combination with nsPEFs, their doses can be significantly reduced into non-toxic ranges.

\section{Future directions for treatment of cancer with nsPEFs}

Nearly all studies with nsPEFs have been conducted in vitro with cells in culture or in vivo as preclinical investigations in mice and rats. In essentially all of these studies, which only a limited number are discussed here, nsPEF ablation has been highly effect against all tumor cells and tissues tested. The in vivo studies reviewed here suggest that clinical trials could most easily begin with skin cancers such as squamous cell carcinoma and/or basal cell carcinoma. This is supported by one example of an nsPEF-treated human basal cell carcinoma from a single patient who had a complete pathological response and the tumor was eliminated [75]. In fact, one human clinical trial treating basal cell carcinoma has been completed, but not yet published [76]. Of course, funding for these trials are difficult to secure and this is presently an issue with such low funding levels and financiers showing caution with investments. The other viable cancer for nsPEF ablation is cancer in internal organs such as HCC or pancreatic tumors. Before these studies begin, catheter electrodes must be developed for laparoscopy approaches which would avoid invasive surgeries. Finally, confirming and defining molecular mechanisms for presumed nsPEF-associated host immune responses will make clinical trials more attractive for physicians and patients and appealing to investors.

\section{Acknowledgements}

The authors would like to thank Dr. Jürgen Kolb, Dr. Shu Xiao and Dr. YeongJer Chen for providing engineering support for these studies. We also thank Drs. Wentia Ford and Wei Ren for their help with various aspects of these studies. The authors also appreciate the technical support from Ms Nova Sain and K. Tyler Harlow. We are also thankful for financial support from Mr. Frank Reidy. These studies also received support in part from an AFOSR/DOD MURI grant on sub cellular responses to narrowband and wideband radio frequency radiation, administered through Old Dominion University and grants from Ethicon Endo Surgery and the Virginia Center for Innovative Technology and the Commonwealth Research Commercialization Fund. The work focuses on studies from the Frank Reidy Research Center for Bioelectrics, at Old Dominion University. We apologize to the many authors in this area, who have done excellent work to advance this field, but were not referenced in this brief, focused review.

\section{References}

1. Richard Restak (2000) Mysteries of the Mind ISBN-10: 0792279417

2. Bowman JS (2000) Columbia Chronologies of Asian History and Culture Columbia University Press. ISBN 9780231110044.

3. Shehata M (2004) The Father of Medicine: A Historical Reconsideration.J Med Ethics12: 171-176.

4. Makara's List of Egyptian Gods.

5. Gods and Mythology of ancient Egypt.

6. Rana RE, Arora BS (2002) History of plastic surgery in India. J Postgrad Med 48: 76-78.

7. Von Staden H (ed.). Herophilos: The Art of Medicine in Early Alexandria Cambridge University Press, 1989 ISBN 0-521-23646-0, ISBN 978-0-52123646-1

8. Longrigg J (1993) Greek Rational Medicine: Philosophy and Medicine from Alcmaeon to the Alexandrians. Psychology Press. ISBN 9780415025942.

9. Sherer A, Epstein F, Constantini S (2004) Hua Tuo, patron of surgeons, or how the surgeon lost his head! Surg Neurol 61: 497-498.

10. Grammaticos PC, Diamantis A (2008) Useful known and unknown views of the father of modern medicine, Hippocrates and his teacher Democritus. Hell J Nucl Med 11: 2-4.

11. Nuland SB (1988) Doctors The Biography of Medicine, Knopf, ISBN 0-39455130-3.

12. Zimmerman LM, Veith I (1993) Great Ideas in the History of Surgery. Norman Publishing. ISBN 9780930405533.
13. Guild WR, Harrison JH, Merrill JP, Murray J (1955) Successful homotransplantation of the kidney in an identical twin. Trans Am Clin Climatol Assoc 67: 167-173

14. FRATER RW, BARNARD CN, SCHRIRE V (1964) OPEN-HEART SURGERY FOR RHEUMATIC DISEASE OF THE MITRAL VALVE: EXPERIENCE AT THE UNIVERSITY OF CAPE TOWN MEDICAL SCHOOL. S Afr Med J 38: 776-781.

15. Kwoh YS, Hou J, Jonckheere EA, Hayati S (1988) A robot with improved absolute positioning accuracy for CT guided stereotactic brain surgery. IEEE Trans Biomed Eng 35: 153-160.

16. Davies BL, Hibberd RD, Ng WS, Timoney AG, Wickham JE (1991) The development of a surgeon robot for prostatectomies. Proc Inst Mech Eng $\mathrm{H}$ 205: 35-38.

17. Cheng JW, Lv Y (2013) New progress of non-surgical treatments for hepatocellular carcinoma. Med Oncol 30: 381.

18. Crocetti L, Lencioni R (2008) Thermal ablation of hepatocellular carcinoma. Cancer Imaging 8: 19-26.

19. Neumann E, Schaefer-Ridder M, Wang Y, Hofschneider PH (1982) Gene transfer into mouse lyoma cells by electroporation in high electric fields. EMBO J 1: 841-845.

20. Daud Al, DeConti RC, Andrews S, Urbas P, Riker Al, et al. (2008) Phase I trial of interleukin-12 plasmid electroporation in patients with metastatic melanoma. J Clin Oncol 26: 5896-5903.

21. Mir LM, Orlowski S, Belehradek J Jr, Paoletti C (1991) Electrochemotherapy potentiation of antitumour effect of bleomycin by local electric pulses. Eur $\mathrm{J}$ Cancer 27: 68-72.

22. Sersa G, Cemazar M, Snoj M (2011) Electrochemotherapy of solid tumorspreclinical and clinical experience. Conf Proc IEEE Eng Med Biol Soc 2011 728-731.

23. Davalos RV, Mir IL, Rubinsky B (2005) Tissue ablation with irreversible electroporation. Ann Biomed Eng 33: 223-231.

24. Miller L, Leor J, Rubinsky B (2005) Cancer cells ablation with irreversible electroporation. Technol Cancer Res Treat 4: 699-705.

25. Pakhomov AG, Phinney A, Ashmore J, Walker K III, Kolb JF, et al. (2004) Characterization of the cytotoxic effect of high-intensity, 10-ns duration electrical pulses. IEEE Trans on Plasma Sci 32: 1579-1586.

26. Nuccitelli R, Pliquett U, Chen X, Ford W, James Swanson R, et al. (2006) Nanosecond pulsed electric fields cause melanomas to self-destruct. Biochem Biophys Res Commun 343: 351-360.

27. Long G, Shires P, Plescia D, Beebe S, Kolb J, et al. (2011) Targeted tissue ablation with nanosecond pulses. IEEE Trans Biomed Eng.

28. Stacey M, Stickley J, Fox P, Statler V, Schoenbach K, et al. (2003) Differentia effects in cells exposed to ultra-short, high intensity electric fields: cell survival, DNA damage, and cell cycle analysis. Mutat Res 542: 65-75.

29. Ibey BL, Roth CC, Pakhomov AG, Bernhard JA, Wilmink GJ, et al. (2011) Dose-dependent thresholds of 10-ns electric pulse induced plasma membrane disruption and cytotoxicity in multiple cell lines. PLoS One 6: e15642.

30. Ren W, Sain NM, Beebe SJ (2012) Nanosecond pulsed electric fields (nsPEFs) activate intrinsic caspase-dependent and caspase-independent cell death in Jurkat cells. Biochem Biophys Res Commun 421: 808-812.

31. Stewart DA, Gowrishankar TR, Weaver JC (2004)Transport lattice approach to describing electroporation: use of local asymptotic model. IEEE Trans Plasma Sci 32: 1696-1708

32. Gowrishankar TR, Esser AT, Vasilkoski Z, Smith KC, Weaver JC (2006) Microdosimetry for conventional and supra-electroporation in cells with organelles. Biochem Biophys Res Commun 341: 1266-1276.

33. Craviso GL, Choe S, Chatterjee P, Chatterjee I, Vernier PT (2010) Nanosecond electric pulses: a novel stimulus for triggering $\mathrm{Ca} 2+$ influx into chromaffin cells via voltage-gated Ca2+ channels. Cell Mol Neurobiol 30: 1259-1265.

34. Morotomi-Yano K, Oyadomari S, Akiyama H, Yano K (2012) Nanosecond pulsed electric fields act as a novel cellular stress that induces translational suppression accompanied by elF2 $\hat{ \pm} \pm$ phosphorylation and 4E-BP1 dephosphorylation. Exp Cell Res 318: 1733-1744.

35. Beebe SJ, Sain NM, Ren W (2013) Induction of Cell Death Mechanisms and Apoptosis by Nanosecond Pulsed Electric Fields (nsPEFs) Cells, in press 
36. Hanahan D, Weinberg RA (2011) Hallmarks of cancer: the next generation. Cell 144: 646-674.

37. Beebe SJ, Fox PM, Rec LJ, Buescher ES, Somers K, Schoenbach KH (2002) Nanosecond Pulsed Electric Field (nsPEF) Effects on Cells and Tissues: Apoptosis Induction and Tumor Growth Inhibition. IEEE Trans Plasma Sci 30 $286-292$.

38. Vernier PT, Ziegler MJ, Sun Y, Gundersen MA, Tieleman DP (2006) Nanoporefacilitated, voltage-driven phosphatidylserine translocation in lipid bilayers--in cells and in silico. Phys Biol 3: 233-247.

39. Beebe SJ, Fox PM, Rec LJ, Willis EL, Schoenbach KH (2003) Nanosecond high-intensity pulsed electric fields induce apoptosis in human cells. FASEB $J$ 17: $1493-1495$

40. Lee RC, Dougherty W (2003) Electrical Injury: Mechanisms, Manifestations and Therapy. IEEE T DielectElectrInsul 10: 810-819.

41. Nuccitelli R, Tran K, Sheikh S, Athos B, Kreis M, et al. (2010) Optimized nanosecond pulsed electric field therapy can cause murine malignant melanomas to self-destruct with a single treatment. Int J Cancer 127: 17271736 .

42. Unpublished clinical trials.

43. Nuccitelli R, Tran K, Athos B, Kreis M, Nuccitelli P, et al. (2012) Nanoelectroablation therapy for murine basal cell carcinoma. Biochem Biophys Res Commun 424: 446-450.

44. Nuccitelli R, Huynh J, Lui K, Wood R, Kreis M, et al. (2013) Nanoelectroablation of human pancreatic carcinoma in a murine xenograft model without recurrence. Int J Cancer 132: 1933-1939.

45. Gish RG, Marrero JA, Benson AB (2010) A multidisciplinary approach to the management of hepatocellular carcinoma. Gastroenterol Hepatol (N Y) 6: 1-16.

46. el-Serag HB (2001) Epidemiology of hepatocellular carcinoma. Clin Liver Dis 5: 87-107, vi.

47. Bruix J, Llovet JM (2002) Prognostic prediction and treatment strategy in hepatocellular carcinoma. Hepatology 35: 519-524.

48. El-Serag HB, Marrero JA, Rudolph L, Reddy KR (2008) Diagnosis and treatment of hepatocellular carcinoma. Gastroenterology 134: 1752-1763.

49. Llovet JM, Brú C, Bruix J (1999) Prognosis of hepatocellular carcinoma: the BCLC staging classification. Semin Liver Dis 19: 329-338.

50. Llovet JM, Fuster J, Bruix J; Barcelona-Clínic Liver Cancer Group (2004) The Barcelona approach: diagnosis, staging, and treatment of hepatocellular carcinoma. Liver Transpl 10: S115-120.

51. Abou-Alfa GK, Schwartz L, Ricci S, Amadori D, Santoro A, et al. (2006) Phase II study of sorafenib in patients with advanced hepatocellular carcinoma. J Clin Oncol 24: 4293-4300.

52. Llovet JM, Ricci S, Mazzaferro V, Hilgard P, Gane E, et al. (2008) Sorafenib in advanced hepatocellular carcinoma. N Engl J Med 359: 378-390.

53. Takehara T, Tatsumi T, Suzuki T, Rucker EB 3rd, Hennighausen L, et al. (2004) Hepatocyte-specific disruption of Bcl-xL leads to continuous hepatocyte apoptosis and liver fibrotic responses. Gastroenterology 127: 1189-1197.

54. Vick B, Weber A, Urbanik T, Maass T, Teufel A, et al. (2009) Knockout of myeloid cell leukemia-1 induces liver damage and increases apoptosis susceptibility of murine hepatocytes. Hepatology 49: 627-636.

55. Hikita H, Takehara T, Shimizu S, Kodama T, Li W, et al. (2009) Mcl-1 and $\mathrm{Bcl}-\mathrm{xL}$ cooperatively maintain integrity of hepatocytes in developing and adult murine liver. Hepatology 50: 1217-1226

56. Friedman SL (2008) Hepatic stellate cells: protean, multifunctional, and enigmatic cells of the liver. Physiol Rev 88: 125-172.

57. Malhi H, Guicciardi ME, Gores GJ (2010) Hepatocyte death: a clear and present danger. Physiol Rev 90: 1165-1194.

58. Canbay A, Friedman S, Gores GJ (2004) Apoptosis: the nexus of liver injury and fibrosis. Hepatology 39: 273-278.
59. Drake N (2011) Forty years on from Nixon's war, cancer research 'evolves' Nat Med 17: 757

60. Chen X, Zhuang J, Kolb JF, Schoenbach KH, Beebe SJ (2012) Long term survival of mice with hepatocellular carcinoma after pulse power ablation with nanosecond pulsed electric fields. Technol Cancer Res Treat 11: 83-93.

61. Zha J, Harada H, Yang E, Jockel J, Korsmeyer SJ (1996) Serine phosphorylation of death agonist $B A D$ in response to survival factor results in binding to 14-3-3 not BCL-X(L) Cell 87: 619-628.

62. Chen X, Kolb JF, Swanson RJ, Schoenbach KH, Beebe SJ (2010) Apoptosis initiation and angiogenesis inhibition: melanoma targets for nanosecond pulsed electric fields. Pigment Cell Melanoma Res 23: 554-563.

63. Bergers G, Benjamin LE (2003) Tumorigenesis and the angiogenic switch. Nat Rev Cancer 3: 401-410.

64. Duff SE, Li C, Garland JM, Kumar S (2003) CD105 is important for angiogenesis evidence and potential applications. FASEB J 17: 984-992.

65. Beebe SJ, Ford WE, Ren W Chen X (2011) Pulse Power Ablation of Melanoma with Nanosecond Pulsed Electric Fields, Treatment of Metastatic Melanoma, Ms Rachael Morton (Ed.), ISBN: 978-953-307-574-7, InTech

66. Nuccitelli R, Tran K, Lui K, Huynh J, Athos B, et al. (2012) Non-therma nanoelectroablation of UV-induced murine melanomas stimulates an immune response. Pigment Cell Melanoma Res 25: 618-629.

67. Kaplan EL, Meier P (1958) Nonparametric estimation from incomplete observations. J Am Stat Assoc 53: 457-481.

68. Tesniere A, Apetoh L, Ghiringhelli F, Joza N, Panaretakis T, et al. (2008) Immunogenic cancer cell death: a key-lock paradigm. Curr Opin Immunol 20 504-511.

69. Green DR, Ferguson T, Zitvogel L, Kroemer G (2009) Immunogenic and tolerogenic cell death. Nat Rev Immunol 9: 353-363.

70. Casares N, Pequignot MO, Tesniere A, Ghiringhelli F, Roux S, et al. (2005) Caspase-dependent immunogenicity of doxorubicin-induced tumor cell death. J Exp Med 202: 1691-1701.

71. Vacchelli E, Galluzzi L, Fridman WH, Galon J, Sautès-Fridman C, et al. (2012) Trial watch: Chemotherapy with immunogenic cell death inducers. Oncoimmunology 1: 179-188

72. Kepp O, Tesniere A, Schlemmer F, Michaud M, Senovilla L, et al. (2009) Immunogenic cell death modalities and their impact on cancer treatment Apoptosis 14: 364-375

73. Wang J, Guo J, Wu S, Feng H, Sun S, et al. (2012) Synergistic effects of nanosecond pulsed electric fields combined with low concentration of gemcitabine on human oral squamous cell carcinoma in vitro. PLoS One 7 e43213.

74. Hall EH, Schoenbach KH, Beebe SJ (2007) Nanosecond pulsed electric fields have differential effects on cells in the S-phase. DNA Cell Biol 26: 160-171.

75. Garon EB, Sawcer D, Vernier PT, Tang T, Sun Y, et al. (2007) In vitro and in vivo evaluation and a case report of intense nanosecond pulsed electric field as a local therapy for human malignancies. Int J Cancer 121: 675-682.

76. Development of a nanosecond pulsed electric field system to treat skin cance Clinicaltrials.gov NCT01463709. 